\title{
Dorsal midline hemivertebra at the lumbosacral junction: report of 2 cases
}

\author{
Shaheryar F. Ansari, MD, ${ }^{1}$ Richard B. Rodgers, MD, ${ }^{2}$ and Daniel H. Fulkerson, MD ${ }^{3}$ \\ 1Department of Neurological Surgery, Indiana University School of Medicine; ${ }^{2}$ Department of Neurological Surgery, Indiana \\ University School of Medicine, Goodman Campbell Brain and Spine; and ${ }^{3}$ Department of Neurological Surgery, Division of \\ Pediatric Neurosurgery, Indiana University School of Medicine, Goodman Campbell Brain and Spine, Indianapolis, Indiana \\ Congenital scoliosis from laterally located hemivertebrae at the lumbosacral junction has been described previously. \\ However, dorsally located midline hemivertebrae at this location have not been reported. The authors describe the \\ presentation, treatment, and outcomes of 2 patients (1 male and 1 female) with this rare malformation. All clinical and \\ radiographic records were reviewed. Outcomes were recorded using survey instruments (Oswestry Disability Index and \\ the 36-Item Short Form Health Survey). Radiographic assessment of bony fusion was performed using CT scanning 1 \\ year after surgery. \\ Both patients presented with back and leg pain, urinary hesitancy/incontinence, difficulty sitting and lying down, waddling \\ gait, and restriction of movement. Imaging showed a wedge-shaped dorsal deformity that stretched the nerve roots and \\ compressed the canal. Both patients underwent resection of the hemivertebra with posterolateral instrumented fusion \\ from L-2 to the pelvis. The female patient had a low-lying conus and underwent sectioning of the filum terminale. Both \\ patients showed improvement in the ability to sit and lie flat and in bowel and bladder function after surgery. The authors \\ describe their experience with 2 patients with similar, rare congenital bony deformities at the lumbosacral junction. To \\ their knowledge, similar cases have not been previously reported.
}

http://thejns.org/doi/abs/10.3171/2014.9.SPINE1411

KEY WORDS dorsal; kyphosis; lumbosacral; hemivertebra; congenital

$\mathrm{L}$ ATERALLY positioned hemivertebrae and the resultant focal scoliosis at the lumbosacral junction have been previously described. ${ }^{1,2,4-8}$ Other authors have described congenital kyphosis from dorsal midline hemivertebrae in the thoracic or upper lumbar spine..$^{9,11,12}$ These previous publications suggest a high risk of deformity progression and neurological decline in patients with congenital kyphosis.

To our knowledge, dorsal midline hemivertebrae causing functional kyphosis at the lumbosacral junction have not been previously reported. We describe 2 patients with radiographically similar lesions. Both patients had a dysplastic L-5 hemivertebra that caused a locally kyphotic defect, stretching the nerves over a wedge-shaped bone. Both patients presented with similar symptoms and improved after surgical treatment. Our surgical strategy and outcomes are described.

\section{Case Reports}

A review of all pertinent medical records began after we received approval from the Indiana University School of Medicine Institutional Review Board. The 2 patients consented to participate in postoperative surveys for outcome assessment. Survey instruments included the Oswestry Disability Index (ODI) and 36-Item Short Form Health Survey (SF-36), outcome tools that have been previously validated in the setting of lumbar pain and surgical outcomes. Both patients have undergone follow-up for at least 2 years after surgery.

\section{Case 1}

History and Examination

This otherwise healthy female patient presented at 17 years of age. She had progressive back pain and noted a 
"lump" in her lower lumbar area that had been present since early childhood. She described difficulty sitting as a result of this protuberance. She had a history of recurrent urinary tract infections for many years.

Upon examination, the patient was a $1.5-\mathrm{m}$ tall, welldeveloped female who walked with a waddling gait, flexed hips, and a compensating exaggerated lumbar lordosis. She had full strength and sensation in her lower extremities. Formal urodynamic studies demonstrated abnormal bladder emptying and capacity.

Imaging demonstrated a midline hemivertebra with a dorsal wedge shape at the lumbosacral junction (Fig. 1). There was focal spinal stenosis with stretching of the nerve roots over the focal kyphotic deformity. There were 4 additional non-rib bearing lumbar vertebrae. The patient had disc spaces above and below the dysplastic vertebra. In addition, her conus appeared low lying, and there was a slight bit of fat apparent in the filum terminale.

\section{Surgical Technique}

Continuous electromyography monitoring and intraoperative stereotactic navigation were used. Screws were placed in the pedicles of L-2 and L-3 and in the ilium bilaterally. Laminectomies of L-4 and the dysplastic L-5 were performed. The pedicles of the L-5 hemivertebra were resected and the L-4, L-5, and S-1 nerve roots were skeletonized (Fig. 2). A disc space was identified above and below the triangular L-5. The hemivertebra was resected by coring the center with a drill and then reducing the dorsal bone into the defect. Bone was then removed using a rongeur.

The thecal sac was significantly stretched over the hemivertebra. It was more relaxed after resection of the dysplastic bone. A midline durotomy was performed. The filum terminale was isolated and tested with a Prass probe.
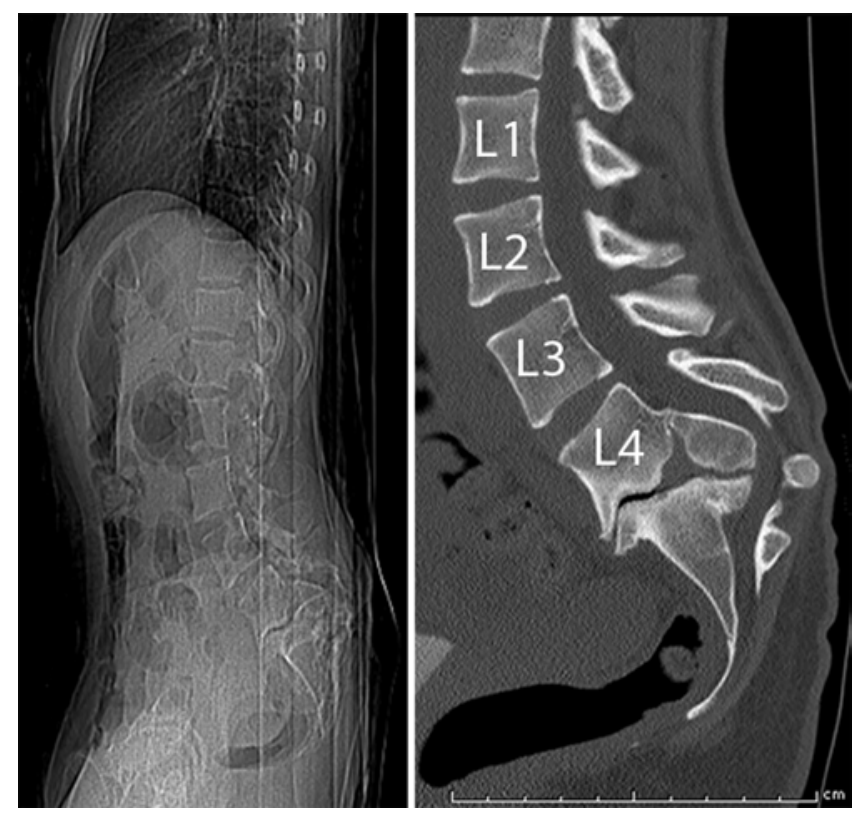

FIG. 1. Case 1. Left: Lateral radiograph demonstrating a bony abnormality at the lumbosacral junction. Right: Sagittal reconstructed CT scan with the non-rib bearing lumbar vertebra labeled. L-5 shows aplasia of the anterior vertebral body with a wedge-shaped posterior.

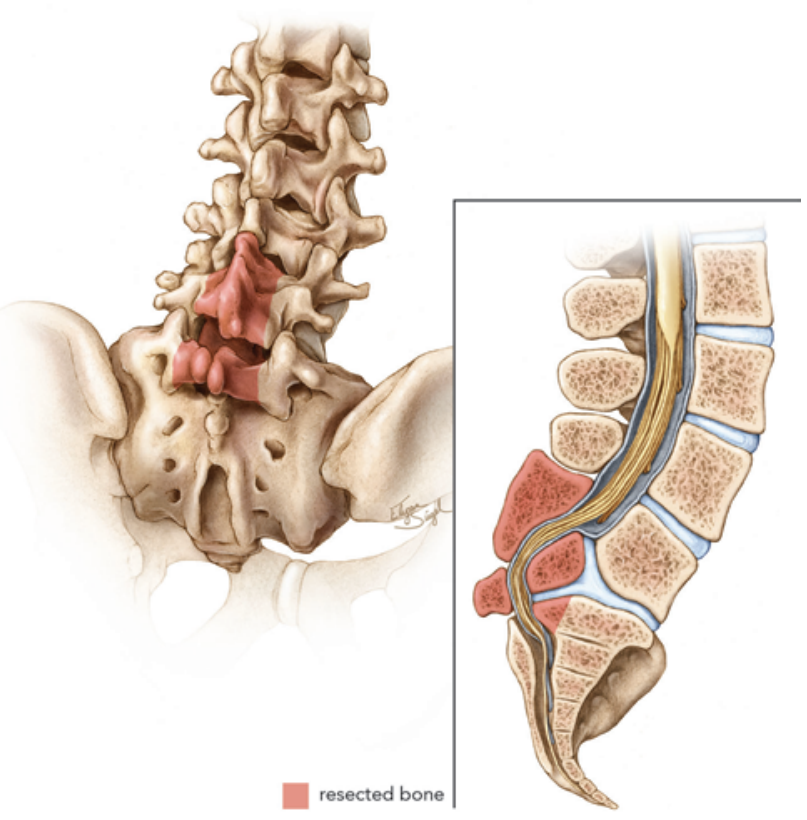

FIG. 2. Case 1. Artist's illustration of the deformity demonstrating the resected bone. Copyright Elyssa Siegel. Published with permission.

After confirming a lack of electrical stimulation, the filum was sectioned. Pathological examination showed that the filum was infiltrated with fat. The durotomy was closed primarily. An artist's rendition of the reconstruction and final decompression is shown in Fig. 3.

After decortication, posterolateral fusion from L-2 to the sacrum was performed using morselized local bone, bone matrix, and recombinant human bone morphogenetic protein-2 (rhBMP-2).

\section{Postoperative Course}

Within 3 months, the patient subjectively reported that she could sit better, walked more upright, and had improved bladder sensation. She remained neurologically intact. CT imaging performed at 1 year demonstrated completed posterolateral bony fusion with improved local canal width (Fig. 4). Her postoperative anteroposterior spinal canal diameter at the L5-S1 level was $14.75 \mathrm{~mm}$, increased from $6.41 \mathrm{~mm}$ before surgery. Figure 5 demonstrates representative preoperative (left) and postoperative (right) axial images.

The patient was quite petite and the heads of the iliac screws protruded. After demonstrating completed fusion, we electively removed all of her hardware. She has returned to full physical activity, including competitive golf.

Survey outcome was obtained 2 years after the initial surgery. The patient reported an ODI score of 6, indicating minimal disability. Her SF-36 scores are listed in Table 1. As shown in the table, her physical functioning is quite high, and she feels no limitations due to her physical, emotional, or social health. She feels her pain is well controlled and her overall health is satisfactory.

\section{Case 2}

History and Examination

This otherwise healthy 31-year-old man was originally 


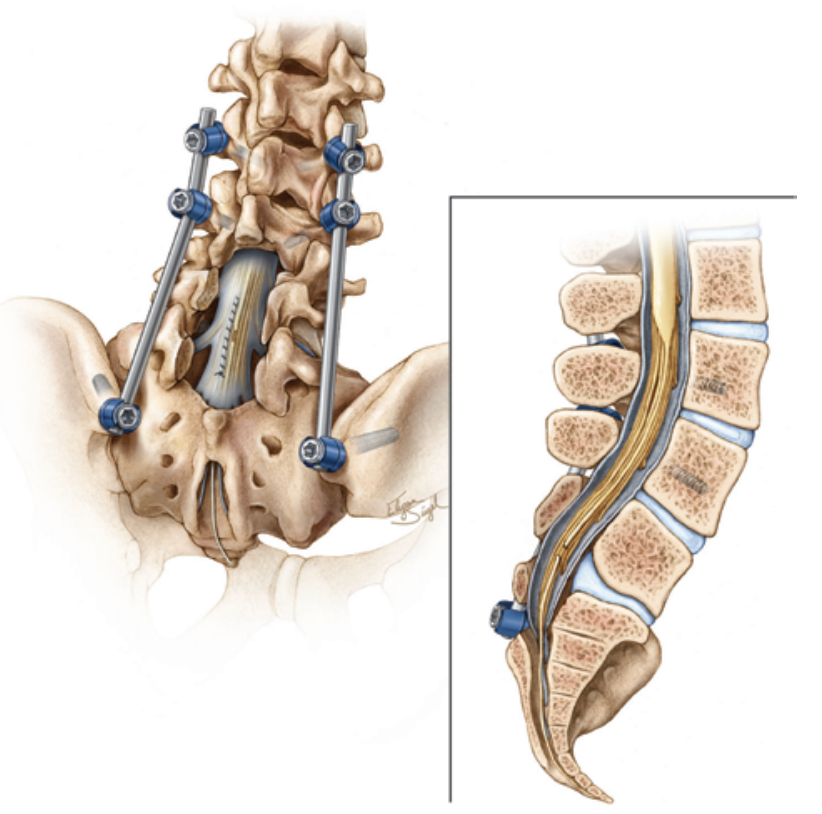

FIG. 3. Case 1. Artist's rendition of the final decompression and reconstruction, including sectioning of the filum terminale. Copyright Elyssa Siegel. Published with permission.

evaluated in childhood for difficulty walking, back pain, and urinary hesitancy. At that time, his defect was deemed "inoperable."

He presented again in adulthood with progressively worsening numbness in his legs and disturbance in his gait. He walked with a waddling gait, an exaggerated lumbar lordosis, and flexed hips. He had been unable to maintain employment and was on disability support. He was a multiple-pack-per-day smoker. He began to develop urinary incontinence approximately 6 months prior to our initial evaluation. He reported difficulty sleeping and he could not lie flat. His physical examination showed some tenderness over a bony mass at the lower lumbar spine. He had some mild weakness in his left dorsiflexor and ex-
TABLE 1. SF-36 scores for both patients

\begin{tabular}{lcc}
\hline \multicolumn{1}{c}{ Category } & Case 1 & Case 2 \\
\hline Physical functioning & 95 & 30 \\
\hline Limitation due to physical health & 100 & 0 \\
\hline Limitations due to emotional problems & 100 & 0 \\
\hline Energy/fatigue & 46 & 50 \\
\hline Emotional well-being & 56 & 52 \\
\hline Social functioning & 100 & 62.5 \\
\hline Pain & 80 & 22.5 \\
\hline General health & 85 & 45 \\
\hline
\end{tabular}

tensor hallucis longus muscles, but he had otherwise full strength in his legs. He did have pain on the straight leg raise test bilaterally. Imaging revealed a dysplastic L4-5 vertebral body with some incorporation of the sacrum (Fig. 6 left). He did not have a defined disc space between the presumed L-4 and L-5, but did have a space between the bony mass and sacrum (Fig. 6 right).

\section{Surgical Technique}

A similar surgical approach was used as in Case 1. We used electromyography and neuronavigation. Screws were placed in L-2 and L-3 and the ilium for posterolateral fusion. The laminae and pedicles of the dysplastic L-5 hemivertebra were resected using a high-speed drill and rongeur. The wedge-shaped posterior deformity was resected. We did not perform a filum section in this case. The posterolateral fusion material consisted of morcellized local bone, bone matrix, and rhBMP-2.

\section{Postoperative Course}

The patient remained neurologically stable with mild left dorsiflexor weakness. He subjectively experienced improvement in his bowel and bladder symptoms. He had improvement in his ability to sit and could lie flat during sleeping. CT scanning at 1 year after surgery showed completed posterolateral bony fusion with resection of the
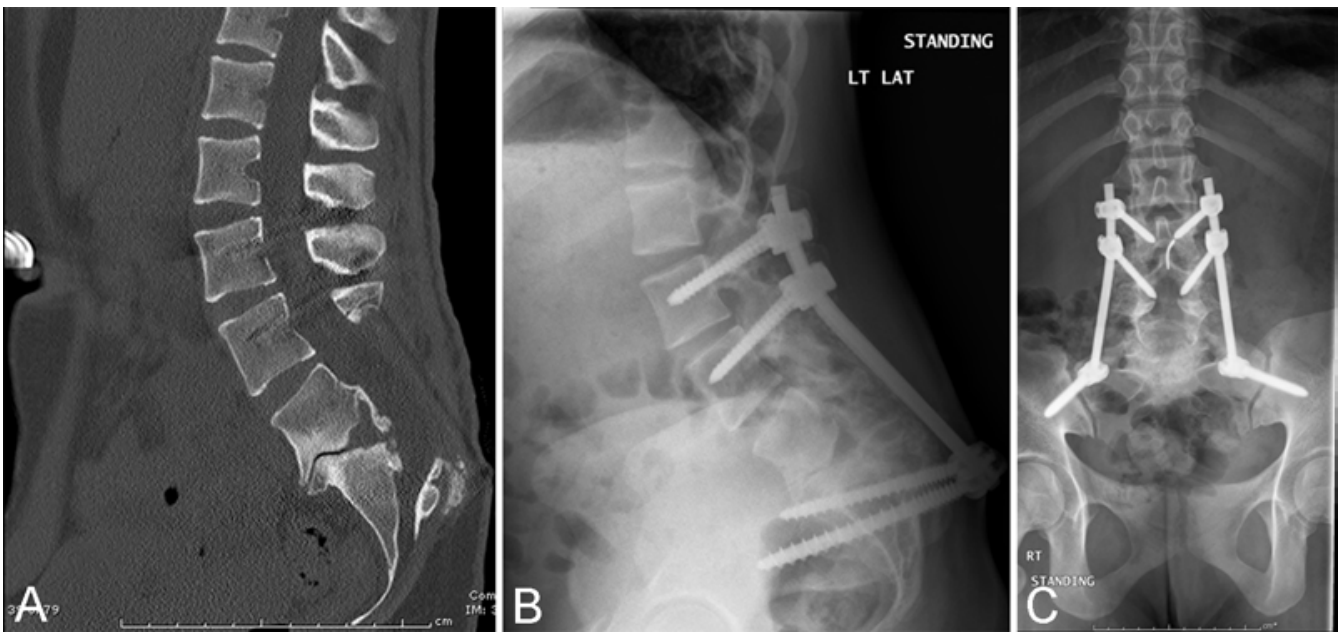

FIG. 4. Case 1. Postoperative sagittal CT (A). Radiographs obtained 1 year after surgery demonstrate the reconstruction and posterolateral fusion in the sagittal $(\mathbf{B})$ and coronal $(\mathbf{C})$ planes. 

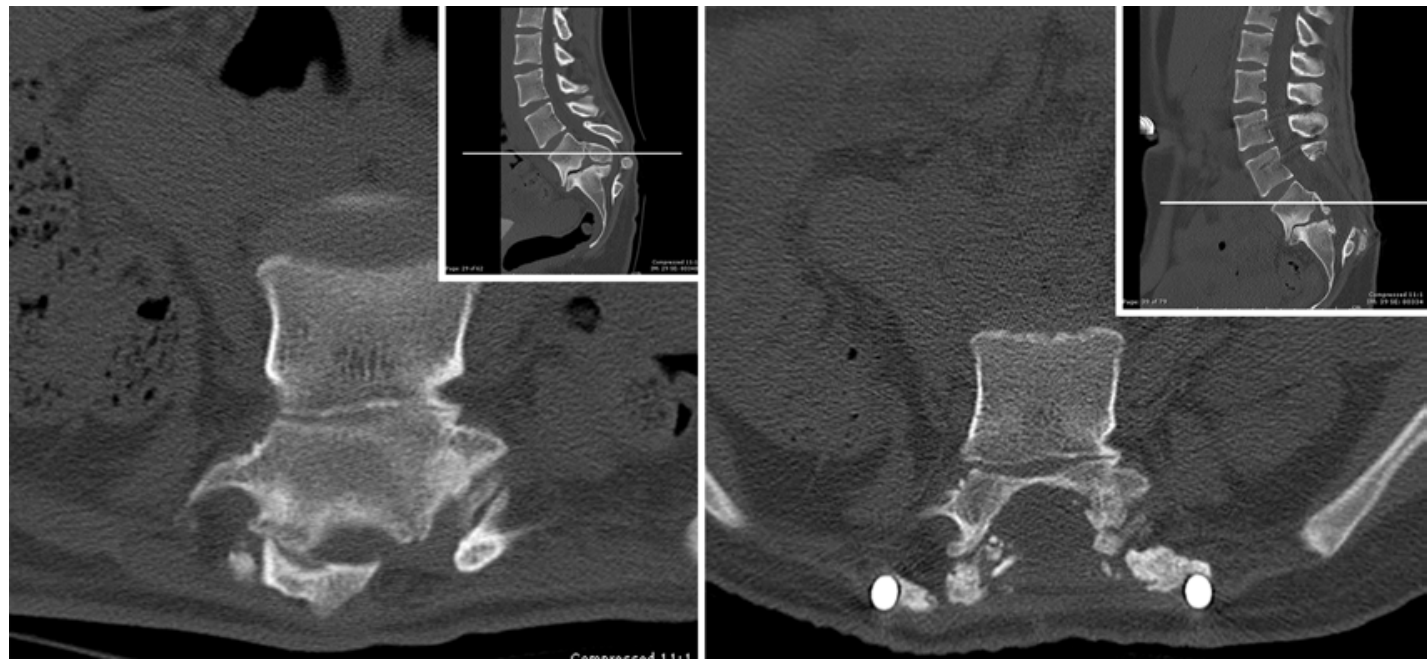

FIG. 5. Case 1. Representative preoperative (left) and postoperative (right) axial images. The insets show the sagittal localization of the axial slice.

dorsal bony wedge (Fig. 7). The anteroposterior diameter of the spinal canal at L5-S1 was increased to $12.8 \mathrm{~mm}$ from $7.6 \mathrm{~mm}$ before surgery. Representative preoperative (Fig. 8 left) and postoperative (Fig. 8 right) axial images are shown.

While the patient reported that he improved, his survey scores 2 years after surgery showed severe disability. He reported an ODI score of 60. His SF-36 scores are listed in Table 1 . He feels significantly impaired in his function due to physical and emotional health issues. He has significant pain and overall does not feel healthy.

\section{Discussion}

The natural history of congenital scoliosis or kyphosis from a hemivertebra has been described previously.,10,12 The risk of progression of the deformity is based on location (higher thoracic lesions carry a worse prognosis) and anatomy. Williams et al. reported progressive deformity associated with loss of function in patients who are treated with observation or bracing alone. ${ }^{9}$ Winter et al. described
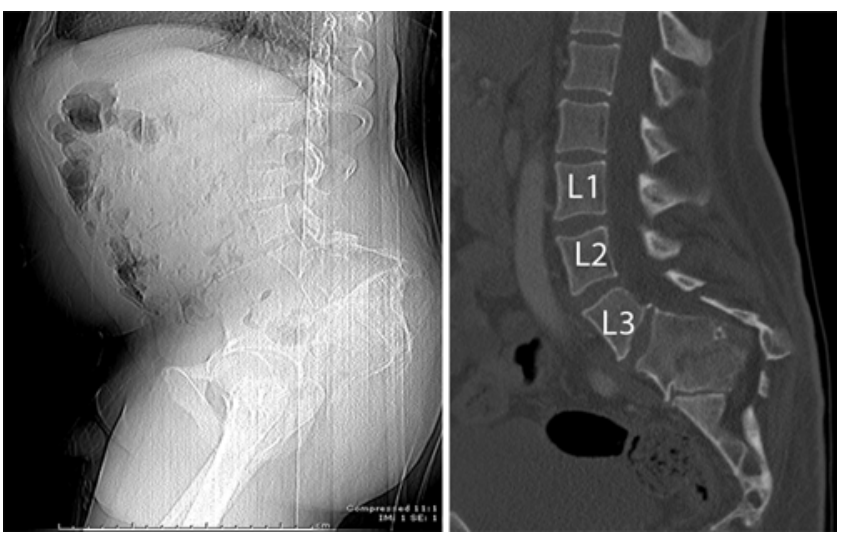

FIG. 6. Case 2. Lateral radiograph (left) and sagittal CT scan (right) showing a similar defect. The non-rib bearing lumbar vertebrae are labeled. an average of $41^{\circ}$ of progression of deformity over 6 years in a group of 130 patients with dorsal hemivertebrae. ${ }^{9}$

Hemivertebrae are classified as Type I (defect in formation), Type II (defect in segmentation), and Type III (mixed)., 3,10 Based on natural history studies, Type I defects have the worst prognosis with progressive deformity and neurological dysfunction., ${ }^{7,-12}$ For progressive congenital kyphosis, Winter and colleagues suggested that laminectomy alone is inadequate, as the cord is often bowstrung across the ventral deformity. ${ }^{10,11}$ In both of our patients, the bones of L-4, L-5, and the sacrum were abnormal, with the most glaring defect apparent in L-5 vertebral body formation (Figs. 1 right and 6 right). The orientation and incomplete separation of L-5 with L-4 and the sacrum indicates a defect in segmentation. Therefore, we categorized both patients as having Type III (mixed) lesions.

Classically, congenital kyphosis from a midline hemivertebra occurs from the orientation of normally shaped vertebral bodies above and below an anterior wedge. In contrast, the anterior longitudinal orientation of our patients was fairly normal. The dysplastic L-5 vertebra was oriented in a wedge dorsally, compressing the spinal canal and stretching the nerve roots. Both patients had 4 nonrib bearing lumbar vertebra above the hemivertebra, with an abnormally shaped sacrum.

The clinical presentation of our patients was similar to those with tethered spinal cord. Both had difficulty sitting and lying flat. Both walked with a waddling gait and flexed hips. Both had bowel and bladder difficulties with abnormal urodynamic testing. The patient in Case 2 was evaluated by both urology and orthopedic surgery as a child, but no surgery was offered at that time. He had significant difficulty throughout his life and was unable to maintain employment. The patient in Case 1 also noted problems since early childhood but was much more functional. Interestingly, both patients felt that their symptoms progressed after skeletal maturity.

Our surgical strategy was similar in both patients. Our goal was to resect the dorsal wedge, thus relaxing the 

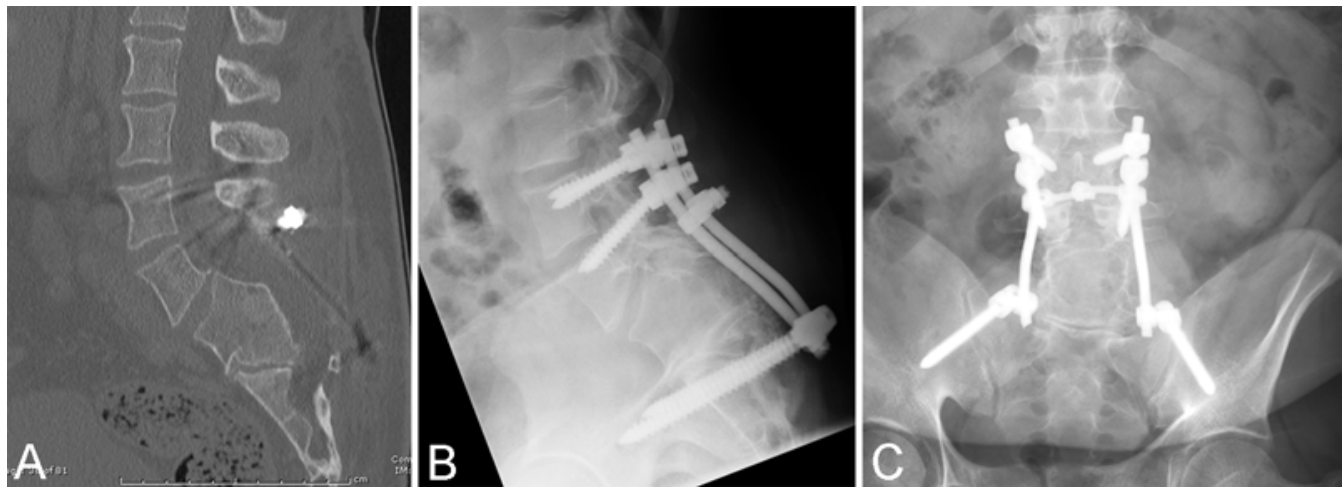

FIG. 7. Case 2. Postoperative images showing the sagittal reconstructed CT (A). The reconstruction is demonstrated on sagittal and coronal radiographs at 1 year after surgery (B and $\mathbf{C})$.

nerve roots. We resected the L- 5 pedicles and the laminae of L-4 and L-5 (Fig. 2). We considered this resection enough to generate instability at the lumbosacral junction. Thus, we performed an instrumented fusion (Fig. 3). The patient in Case 1 had a low-lying conus with a fatty filum. We elected to perform a filum section. We did not perform a filum section in the other patient (Case 2) as his conus appeared normal.

Both patients improved in their ability to sit and lie flat, and posture was improved in both cases. Both patients reported improvement in bowel and bladder function. The patient in Case 1 recovered well with minimal disability as exhibited by her ODI scores. She has full function and minimal residual pain. The other patient (Case 2) reported improvement in his ability to walk, sit, and lie flat. He also reported improvement in bowel and bladder function. However, he continues to have significant back and leg pain, with ODI scores that indicate severe disability.

\section{Conclusions}

We describe our experience and the outcomes of 2 pa-
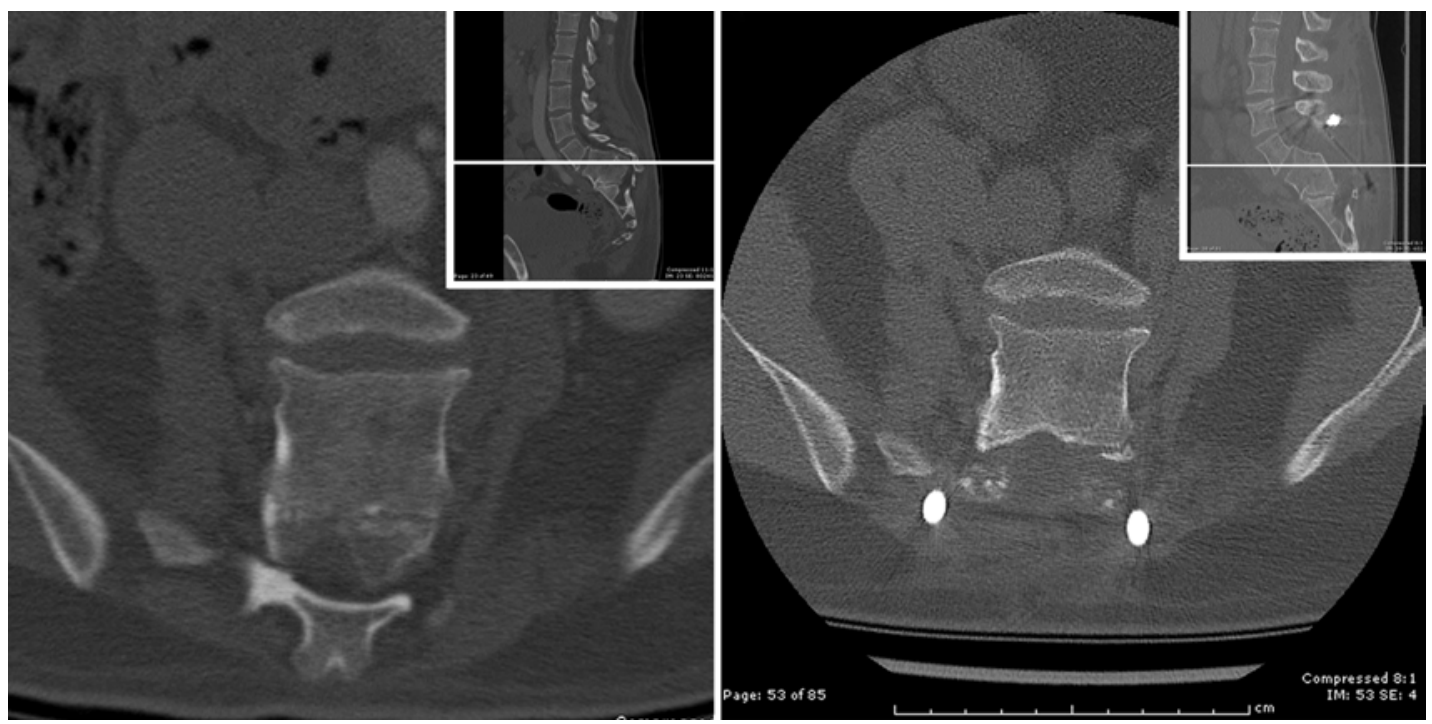

FIG. 8. Case 2. Representative preoperative (left) and postoperative (right) axial images. The insets show the sagittal localization of the axial slice. 
approach: innovative operative technique and first results. Spine (Phila Pa 1976) 27:1116-1123, 2002

6. Ruf M, Jensen R, Letko L, Harms J: Hemivertebra resection and osteotomies in congenital spine deformity. Spine (Phila Pa 1976) 34:1791-1799, 2009

7. Slabaugh PB, Winter RB, Lonstein JE, Moe JH: Lumbosacral hemivertebrae. A review of twenty-four patients, with excision in eight. Spine (Phila Pa 1976) 5:234-244, 1980

8. Suk SI, Chung ER, Lee SM, Lee JH, Kim SS, Kim JH: Posterior vertebral column resection in fixed lumbosacral deformity. Spine (Phila Pa 1976) 30:E703-E710, 2005

9. Williams F, McCall IW, O'Brien JP, Park WM: Severe kyphosis due to congenital dorsal hemivertebra. Clin Radiol 33:445-452, 1982

10. Winter RB: Congenital kyphosis. Clin Orthop Relat Res (128):26-32, 1977

11. Winter RB, Moe JH, Wang JF: Congenital kyphosis. Its natural history and treatment as observed in a study of one hundred and thirty patients. J Bone Joint Surg Am 55:223-256, 1973
12. Zidorn T, Krauspe R, Eulert J: Dorsal hemivertebrae in children's lumbar spines. Spine (Phila Pa 1976) 19:2456-2460, 1994

\section{Author Contributions}

Conception and design: Fulkerson, Ansari. Acquisition of data: all authors. Analysis and interpretation of data: Fulkerson. Drafting the article: all authors. Critically revising the article: Fulkerson. Reviewed submitted version of manuscript: Fulkerson. Approved the final version of the manuscript on behalf of all authors: Fulkerson. Study supervision: Fulkerson.

\section{Correspondence}

Daniel H. Fulkerson, Indiana University School of Medicine/ Goodman Campbell Brain and Spine, Riley Hospital for Children, 702 Barnhill Dr. \#1134, Indianapolis, IN 46202. email: dfulkers @iupui.edu. 